\title{
Net clinical benefit of non-vitamin K antagonist oral anticoagulants in atrial fibrillation and chronic kidney disease: a trade-off analysis from four phase III clinical trials
}

\author{
Zhi-Chun Gu ${ }^{1 \#}$, Ling-Cong Kong ${ }^{2 \#}$, Shuo-Fei Yang ${ }^{3}$, An-Hua Wei ${ }^{4}$ Na Wang ${ }^{5}$, Zheng Ding ${ }^{6}$, Chi Zhang ${ }^{1}$, \\ Xiao-Yan Liu ${ }^{1}$, Ying-Li Zheng ${ }^{6}$, Hou-Wen Lin ${ }^{1}$ \\ ${ }^{1}$ Department of Pharmacy, ${ }^{2}$ Department of Cardiology, ${ }^{3}$ Department of Vascular Surgery, Renji Hospital, School of Medicine, Shanghai Jiaotong \\ University, Shanghai 200127, China; ${ }^{4}$ Department of Pharmacy, Tongji Hospital, Tongji Medical College, Huazhong University of Science and \\ Technology, Wuhan 430030, China; ${ }^{5}$ Department of Pharmacy, The Second Affiliated Hospital of Chongqing Medical University, Chongqing \\ 400010, China; ${ }^{\circ}$ Department of Pharmacy, Fuwai Hospital, Chinese Academy of Medical Sciences, Beijing 100037, China \\ Contributions: (I) Conception and design: ZC Gu, LC Kong; (II) Administrative support: SF Yang, YL Zheng, HW Lin; (III) Provision of \\ study materials or patients: AH Wei, N Wang, Z Ding, C Zhang; (IV) Collection and assembly of data: ZC Gu, XY Liu; (V) Data analysis and \\ interpretation: ZC Gu; (VI) Manuscript writing: All authors (VII) Final approval of manuscript: All authors. \\ \#These authors contributed equally to this work as first authors. \\ Correspondence to: Shuo-Fei Yang, MD, PhD. Renji Hospital, School of Medicine, Shanghai Jiaotong University, Shanghai 200127, China. \\ Email: doctor_yangshuofei@163.com; Ying-Li Zheng, MD. Department of Pharmacy, Fuwai Hospital, Chinese Academy of Medical Sciences, \\ Beijing 100037, China. Email: zhengyl1625@sina.com.
}

Background: Atrial fibrillation (AF) is quite prevalent in patient with chronic kidney disease (CKD). This study mainly investigated the net clinical benefit (NCB) property of non-vitamin K antagonist oral anticoagulants (NOACs) versus warfarin in patients with $\mathrm{AF}$ and CKD by a pooled-analysis.

Methods: A comprehensive search of Medline, Embase, Cochrane Library and Clinical Trials.gov Website was performed for eligible randomized controlled trials (RCTs) reporting the efficacy and safety outcomes according to renal function of NOACs. Pre-specified outcomes and their number of patients needed to treat (NNT), including stroke/systemic embolism (SSE), major bleeding, and all-cause death, were evaluated using a random-effects model. NCB that balanced SSE and major bleeding was calculated using Singer's method.

Results: Four phase III clinical trials including 70,952 patients were enrolled, 45,265 (64\%) with CKD, and 25,687 (36\%) without CKD; 41,942 (59\%) taking NOACs and 29,010 (41\%) taking warfarin. Risks of SSE [relative risk (RR): 0.80, 95\% confidence interval (CI): 0.73-0.88, $\mathrm{P}<0.01$ ], major bleeding (RR: 0.79, 95\% CI: 0.66-0.96, P=0.017), and all-cause death (RR: 0.91, 95\% CI: 0.84-0.99, P=0.031) were significantly lower in CKD patients with NOACs than those with warfarin, accompanying with a high absolute risk reduction (NNT: 182 for SSE; 122 for major bleeding; 196 for all-cause death). While NOACs were not superior to warfarin on SSE, major bleeding, and all-cause death in patients without CKD, the NCB of NOACs versus warfarin was progressively increased with the deterioration of renal function (NCB: 0.72 for no CKD, 1.59 for mild CKD, 2.74 for moderate CKD). Sensitivity analyses did not significantly affect the primacy results.

Conclusions: NOACs, compared with warfarin, provide a better clinical profile on SSE, major bleeding, all-cause death, and NCB in CKD patients.

Keywords: Atrial fibrillation (AF); chronic kidney disease (CKD); oral anticoagulants; non-vitamin K antagonist oral anticoagulants (NOACs); net clinical benefit (NCB)

Submitted Apr 17, 2019. Accepted for publication Jun 20, 2019.

doi: $10.21037 / \mathrm{cdt} .2019 .07 .09$

View this article at: http://dx.doi.org/10.21037/cdt.2019.07.09 


\section{Introduction}

The prevalence of atrial fibrillation (AF) is high in patients with chronic kidney disease (CKD) (1). CKD shows an increased risk for cardiovascular disease, meanwhile is itself an important predictor of thrombosis and hemorrhage (2). Additionally, CKD is widely regarded as a risk factor for the low time in therapeutic range (TTR), and superimposed platelet dysfunction in AF participants treated with warfarin (3). Therefore, AF patients with the morbid state of CKD are even at an increased risk for stroke or systemic embolism and hemorrhage.

Non-vitamin K antagonist oral anticoagulants (NOACs), with favorable efficacy and safety profiles, confer practical advantages in AF (4). In 2018, data from Taiwan showed that NOACs accounted for about $73 \%$ of overall OACs prescribed for patients with incident AF (5). Interestingly, regardless of the limited evidence in fragile patients, the usage of NOACs in CKD patients is still increasing (6). NOACs mainly depend on some degree of renal excretion (dabigatran $80 \%$; edoxaban $50 \%$; rivaroxaban $36 \%$; apixaban $27 \%$ ) (7). Thus, their pharmacokinetics properties may be effected by renal function impairment, and bring about consequent increase in free drug levels in the blood (8). Currently, there hasn't been any randomized clinical trials (RCTs) conducted yet, to assess the efficacy and safety of NOACs in AF patients with CKD. Nielsen $e t$ $a l$. and Andò $e t a l$. assessed the efficacy and safety of NOACs across CKD subgroup, but only individual NOACs results were available for their study $(9,10)$. Sardar et al. and DelCarpio Munoz et al. reported a marked reduction of SSE and major bleeding of NOACs compared to that of warfarin in patients with CKD, but number of patients needed to treat (NNT) and net clinical benefit (NCB) was not detected $(11,12)$. Of note, the concept of NCB has been developed and gradually used to quantify the balance between a reduced risk of SSE and an increased risk of bleeding with oral anticoagulants in AF during recent years (13). Whereas, evidence on NCB of AF was derived mainly from registry-based cohorts and observational studies, data from RCTs, especially for patients with CKD and AF is quite limited (14-16). Thus, we mainly evaluated the NCB property of NOACs in patients with AF and CKD by a pooled analysis and detect the difference of $\mathrm{NCB}$ on NOACs in varying degrees of renal function.

\section{Methods}

\section{Data sources and search strategy}

The study was performed according to the standards of the Preferred Reporting Items for Systematic Reviews and Meta-Analyses (PRISMA) statement and the Cochrane Handbook (PROSPERO number: CRD42019116940). Medline, Embase, and Cochrane Library were searched from inception to Jan 25, 2019, for identifying all potential studies. For the subject term 'NOACs', the following terms were included: 'Pradaxa' OR 'dabigatran' OR 'Xarelto' OR 'rivaroxaban' OR 'Eliquis' OR 'apixaban' OR 'Savaysa' OR 'edoxaban' OR 'Bevyxxa' OR 'betrixaban' OR 'Nonvitamin $\mathrm{K}$ antagonist oral anticoagulants' $\mathrm{OR}$ 'direct oral anticoagulants' or 'NOACs' OR 'DOACs' OR 'new oral anticoagulants' OR 'factor Xa inhibitors' OR 'novel oral anticoagulants' OR 'factor II a inhibitors'. For the subject term 'atrial fibrillation', the terms were 'AF' OR 'atrial fibrillation'. For the subject term 'RCTs', the following terms were included: 'clinical trial' OR 'controlled clinical trial' OR 'randomized controlled trial'. The Boolean operator 'AND' was used to combine three search themes. Additionally, we identified unpublished trials from the ClinicalTrials.gov Website. The eligibility of each relevant study was independently assessed by two reviewers (ZhiChun Gu and Ling-Cong Kong), with any disagreements being disposed by a third author (Shuo-Fei Yang).

\section{Study selection}

The studies must met the following inclusion and exclusion criteria: (I) study were phase III RCTs of AF patients and taking NOACs in comparison to warfarin; (II) study reporting data about renal function and associated and detailed outcomes; (III) RCTs including patients with mitral stenosis or prosthetic cardiac valves, mean or median follow-up $<6$ months, $<200$ subjects, and NOAC phase II studies were excluded. If studies reporting over one publication, data were retrieved from the most complete publication, we also obtained the other reports for clarifying or complementing the information. Two reviewers (ZhiChun Gu and Ling-Cong Kong) independently evaluated study titles and abstracts to determine the eligibility, then full paper was obtained and evaluated the feasibility in line 
with the inclusion criteria. A third author (Shuo-Fei Yang) resolved all discrepancies and uncertainties.

\section{Study outcomes}

Pre-specified outcomes for the current analysis were stroke and systemic embolism (SSE), major bleeding, allcause death, and calculated NCB. The definition of major bleeding is followed by International Society of Thrombosis and Hemostasis (ISTH). All the data were classified as no CKD group (creatinine clearance- $\mathrm{CrCl}>80 \mathrm{~mL} / \mathrm{min}$ ), mild $\mathrm{CKD}(\mathrm{CrCl}=50-80 \mathrm{~mL} / \mathrm{min})$, and moderate $\mathrm{CKD}(\mathrm{CrCl}$ $30-50 \mathrm{~mL} / \mathrm{min}$ ), using the intention-to-treat principle. The $\mathrm{CrCl}$ was calculated by the Cockcroft-Gault formula.

\section{Data extraction and quality evaluation, and bias assessment}

Information were extracted by two reviewers independently using a priori designed form, including publication year, follow up duration, number of patients, mean age, sex, $\mathrm{AF}$ type, mean CHADS2 score, risk factors, and prior medicine use. In ARISTOTLE, moderate CKD was defined as $\mathrm{CrCl}$ $25-50 \mathrm{~mL} / \mathrm{min}$ (17). The methodological quality of studies was assessed in line with the Cochrane Collaboration Risk of Bias Tool, which include random sequence generation, allocation concealment, blinding, incomplete outcome data, selective reporting, and other biases (18). When more than 10 studies were included, the visual inspection of funnel plots was used to explore potential publication bias (18).

\section{Data analysis}

As mentioned above, subgroup data was abstracted as no CKD group, mild CKD group, and moderate CKD group, which did not provide combined data of CKD $(\mathrm{CrCl}<80 \mathrm{~mL} / \mathrm{min})$. Therefore, data of mild CKD group and moderate CKD group was combined as a one camp. In addition, 2 different dosages of NOACs (edoxaban 30 and $60 \mathrm{mg}$ once daily in ENGAGE AF-TIMI 48, and dabigatran 110 and $150 \mathrm{mg}$ twice daily in RE-LY) were in comparison with warfarin. Similarly, data of different dosages was merged as a one camp.

Relative risks (RRs) and 95\% confidence intervals (CIs) of the outcomes in NOACs versus warfarin were calculated using a random-effects model. The heterogeneity was evaluated through $\mathrm{I}^{2}$ test and Q statistic. P value of $<0.05$ at $\mathrm{Q}$ statistic indicated a significant heterogeneity, and $\mathrm{I}^{2}$ of $>50 \%$ represented considerable heterogeneity (19). The number of patients needed to treat (NNT) to prevent 1 event was calculated as: $(1 /$ absolute risk reduction $) \times 100$, where absolute risk reduction was rate difference (event rates per 100 patients-year on warfarin minus event rates per 100 patients-year on NOACs) (14). Compared to warfarin, the NCB of NOACs was calculated following the formula: (rate of SSE on warfarin minus the rate of SSE on NOACs) - weight $\times$ (rate of major bleeding on NOACs minus rate of major bleeding on warfarin), where rate was event rates per 100 patients-year. We assigned the weighting factor of 1.5 , and provided extra sensitivity analysis using 1.0 and 2.0 weighted factor (13). Because potential effect modifiers (demographic characteristics, risk factors, and prior medicine use) may lead to bias on outcomes of stroke/ SE and major bleeding, a meta-regression analysis was performed to explore the outcomes influenced by these factors. Because original data of moderate CKD and mild CKD was combined as a one group, sensitivity analysis was performed in moderate CKD and mild CKD, respectively. Furthermore, additional analyses were performed to explore the influence by removing the low-dose arms (dabigatran $110 \mathrm{mg}$ in RE-LY, and edoxaban $30 \mathrm{mg} / 15 \mathrm{mg}$ in ENGAGE AF-TIMI 48). Meanwhile, interaction analysis was used for identifying the discrepancies in groups of different renal function. The statistical analyses were conducted by STATA software (version13, Statacorp, College Station, Texas, USA), $\mathrm{P}$ value $<0.05$ was considered statistically significant.

\section{Results}

\section{Study summary}

The flowchart of references was presented in Figure S1. We initially screened 3,244 records, with 842 duplicates. A total of 2,248 articles were excluded by reviewing the titles and abstracts. The remained 154 full texts were obtained, 46 texts met the inclusion criteria, but only 6 studies provided the original data. J-ROCKET AF was excluded from the analysis due to the limited population representation (only Japanese). Due to the use of aspirin, not warfarin, as a comparison, we excluded the AVERROES study from the analysis. Eventually, four phase III trials, comparing NOACs to warfarin in $\mathrm{AF}$ patients, were included in the current analyses: the RE-LY (Randomized Evaluation of Long-term Anticoagulation Therapy) trial, comparing dabigatran with warfarin (20); the ROCKET AF (Rivaroxaban Once Daily Oral Direct Factor Xa Inhibition Compared with Vitamin 


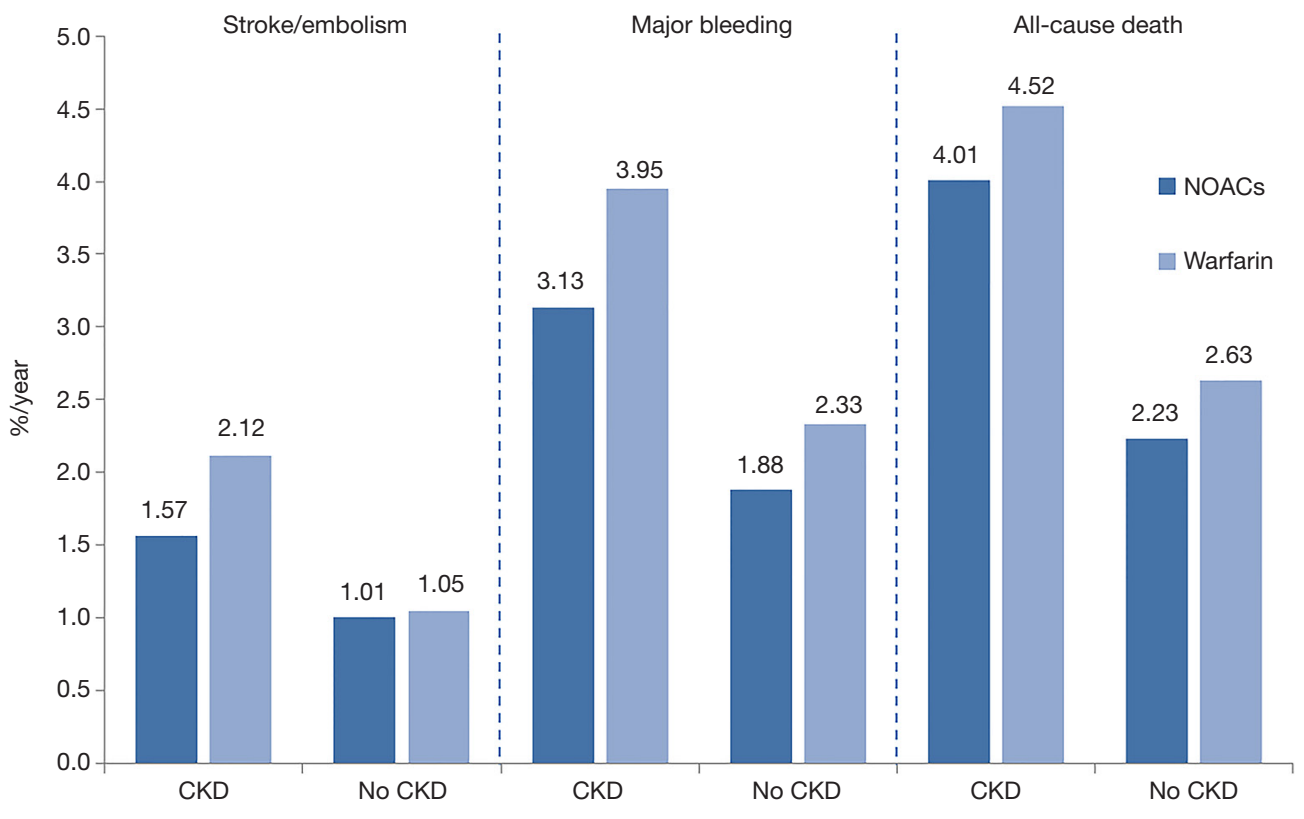

\begin{tabular}{|c|c|c|c|c|c|c|}
\hline Total $(n)$ & 45,265 & 25,687 & 45,319 & 25,706 & 22,711 & 13,362 \\
\hline NOACs events $(n)$ & 881 & 340 & 1,595 & 525 & 1,140 & 339 \\
\hline Warfarin events $(n)$ & 766 & 235 & 1,326 & 457 & 859 & 292 \\
\hline
\end{tabular}

Figure 1 Pooled rates of outcomes in patients with and without CKD. Rate is expressed as \%/year. NOACs, non-vitamin K antagonist oral anticoagulants; CKD, chronic kidney disease.

K Antagonism for Prevention of Stroke and Embolism Trial in Atrial Fibrillation) trial, comparing rivaroxaban to warfarin (21); ARISTOTLE (Apixaban for Reduction in Stroke and Other Thromboembolic Events in Atrial Fibrillation) trial, comparing apixaban to warfarin (17); ENGAGE AF-TIMI 48 (Effective Anticoagulation with Factor Xa Next Generation in Atrial Fibrillation) trial, comparing edoxaban to warfarin (22).

As shown in Figure 1, 70,952 patients were included in the analyses, 45,265 with CKD, of whom 26,770 were treated with NOACs and 18,495 with warfarin, while 25,687 without CKD, of whom 15,172 treated with NOACs and 10,515 with warfarin. A total of 881 SSEs (1.57\%/year) in NOACs-treated patients with CKD and 340 SSEs (1.01\%/year) in NOACs-treated patients without CKD were reported. Similarly, 1,595 major bleedings (3.13\%/year) in NOACs-treated patients with CKD and 525 major bleedings (1.88\%/year) in NOACs-treated patients without CKD were reported. Characteristics of subjects with CKD were shown in Table 1. The duration of median follow-up ranged from 1.5 to 2.8 years across four trials. Except for the RE-LY, which was not double-blinded, all trials met the needs of bias tool items. The included studies had low bias overall and thus had high quality for our investigation (Table S1).

\section{NOACs versus warfarin in patients with CKD and without CKD}

In patients with CKD, NOACs demonstrated a significantly decreased risk for SSE by $20 \%$ (RR: 0.80, 95\% CI: 0.73-0.88, $\mathrm{P}<0.01, \mathrm{I}^{2}: 0.0 \%$ ), major bleeding by $21 \%$ (RR: $0.79,95 \%$ CI: $\left.0.66-0.96, \mathrm{P}=0.017, \mathrm{I}^{2}: 85.3 \%\right)$, and all-cause death by 9\% (RR: $0.91,95 \%$ CI: $0.84-0.99, \mathrm{P}=0.031, \mathrm{I}^{2}: 0.0 \%$ ) when compared with warfarin (Figure $2 A$ ). However, in patients without CKD, SSE risk did not significantly differ in patients treated with NOACs and warfarin (RR: $0.99,95 \%$ CI: $0.75-$ $1.32, \mathrm{P}=0.96, \mathrm{I}^{2}: 64.8 \%$ ). The risks of major bleeding (RR: 0.80, 95\% CI: $\left.0.58-1.09, \mathrm{P}=0.15, \mathrm{I}^{2}: 84.2 \%\right)$ and all-cause death (RR: $0.87,95 \%$ CI: $0.74-1.01, \mathrm{P}=0.07, \mathrm{I}^{2}: 0.0 \%$ ) were numerically lower in NOACs arm, but showed no statistical significance (Figure 2B). No differences in treatment effect 
Table 1 Baseline characteristics of included trials by CKD

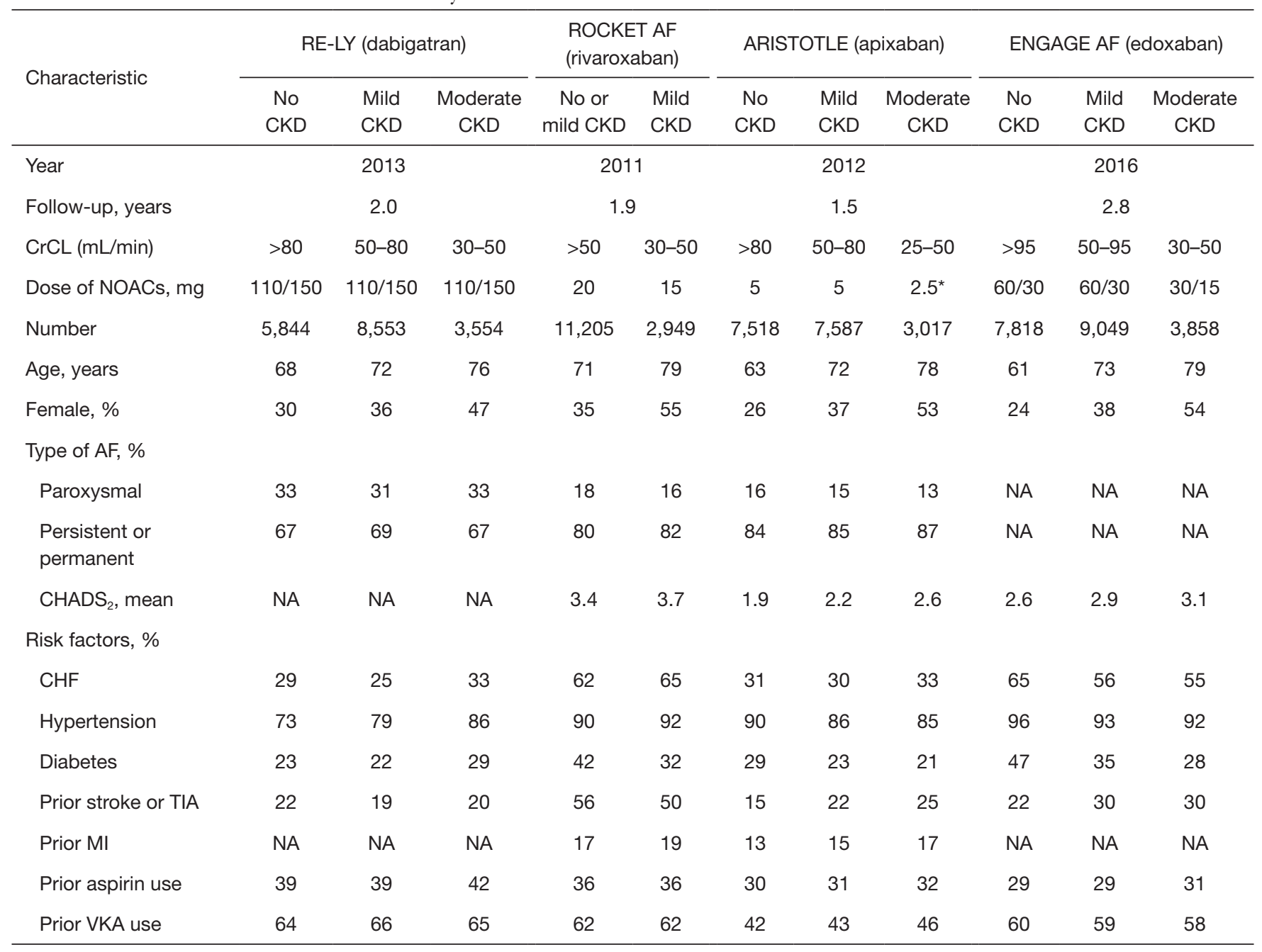

*, apixaban $2.5 \mathrm{mg}$ twice daily is used for patients with serum creatinine $>1.5 \mathrm{mg} / \mathrm{dL}$. AF, atrial fibrillation; CKD, chronic kidney disease; CHF, congestive heart failure; CrCL, creatinine clearance; MI, myocardial infarction; NA, not available; TIA, transient ischemic attack; VKA, vitamin K antagonist; RE-LY, randomized evaluation of long-term anticoagulation therapy; ROCKET AF, rivaroxaban once daily oral direct factor Xa inhibition compared with vitamin $\mathrm{K}$ antagonism for prevention of stroke and embolism trial in atrial fibrillation; ARISTOTLE, apixaban for reduction in stroke and other thromboembolic events in atrial fibrillation; ENGAGE AF, effective anticoagulation with factor Xa next generation in atrial fibrillation.

were found between CKD and no CKD arms on SSE $\left(\mathrm{P}_{\text {interaction }}=0.206\right)$, major bleeding $\left(\mathrm{P}_{\text {interaction }}=0.947\right)$, and allcause death $\left(\mathrm{P}_{\text {interaction }}=0.612\right)$ (Figure $\left.S 2\right)$.

\section{NNT and NCB of NOACs in patients with CKD and without CKD}

In patients with $\mathrm{CKD}$, the event rates per 100 patientsyear of SSE were 1.57 for NOACs, and 2.12 for warfarin, which was translated to NNT of 182, indicating that 182
NOACs patients could prevent 1 SSE event per year than warfarin patients (Table 2). Similarly, the NNT of major bleeding was 122, and the NNT of all-cause death was 196 . In patients without CKD, all the NNTs of outcome were positive (2,500 for SSE, 222 for major bleeding, and 250 for all-cause death, respectively). Thus, the overall beneficial effect of NOACs compared with warfarin to prevent 1 SSE, major bleeding, or all-cause death would be greater in patients with CKD than those without CKD, with a smaller NNT. The NCB analyses stratified by CKD were presented 

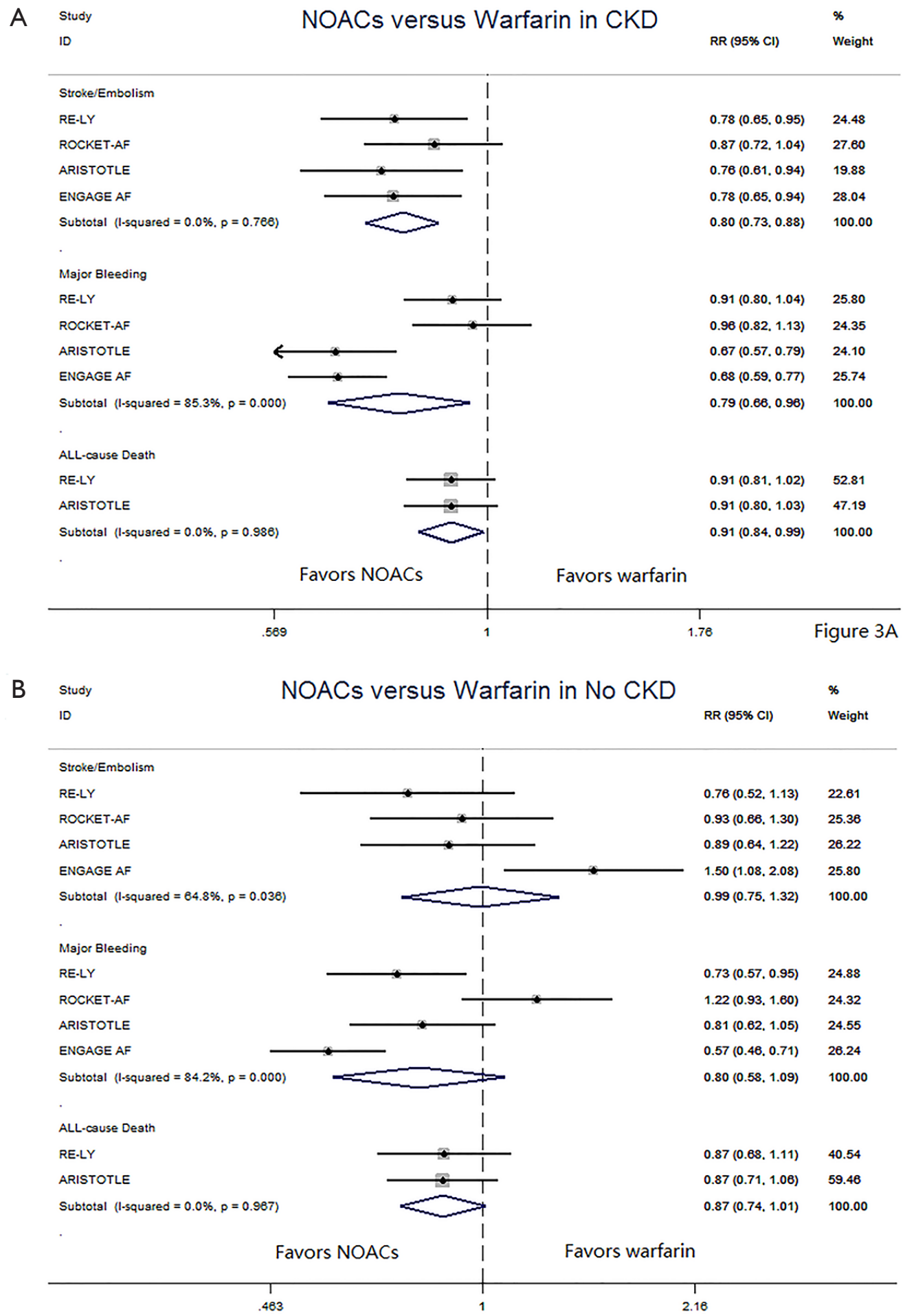

Figure 2 Outcomes of NOACs versus warfarin in patients with (A) and without CKD (B). NOACs, non-vitamin K antagonist oral anticoagulants; CKD, chronic kidney disease; RR, relative risk; 95\% CI, 95\% confidence interval. 
Table 2 Event rates and number needed to treat of NOAC versus Warfarin

\begin{tabular}{|c|c|c|c|c|}
\hline Outcome & $\begin{array}{l}\text { NOAC } \\
\text { (\%/year) }\end{array}$ & $\begin{array}{l}\text { Warfarin } \\
\text { (\%/year) }\end{array}$ & ARR & NNT \\
\hline \multicolumn{5}{|l|}{ Stroke/embolism } \\
\hline Moderate CKD & 2.13 & 2.66 & 0.53 & 189 \\
\hline Mild CKD & 1.39 & 1.96 & 0.57 & 175 \\
\hline CKD & 1.57 & 2.12 & 0.55 & 182 \\
\hline No CKD & 1.01 & 1.05 & 0.04 & 2500 \\
\hline \multicolumn{5}{|l|}{ Major bleeding } \\
\hline Moderate CKD & 3.92 & 5.39 & 1.47 & 68 \\
\hline Mild CKD & 2.88 & 3.56 & 0.68 & 147 \\
\hline CKD & 3.13 & 3.95 & 0.82 & 122 \\
\hline No CKD & 1.88 & 2.33 & 0.45 & 222 \\
\hline \multicolumn{5}{|l|}{ All-cause death } \\
\hline Moderate CKD & 7.13 & 7.86 & 0.73 & 137 \\
\hline Mild CKD & 3.36 & 3.91 & 0.55 & 182 \\
\hline CKD & 4.01 & 4.52 & 0.51 & 196 \\
\hline No CKD & 2.23 & 2.63 & 0.40 & 250 \\
\hline
\end{tabular}

ARR, absolute rate reduction; CKD, chronic kidney disease; NOAC, novel oral anticoagulant; NNT, number needed to treat; $\% /$ year, events per 100 patients-years. in Figure 3. When using a weighted factor of 1.5, NOACs had a positive NCB in patients with CKD when compared with warfarin, yielding an NCB of 1.78 (95\% CI: 1.29-2.27, $\mathrm{P}<0.01)$. Similarly, in patients without CKD, NOACs had superior $\mathrm{NCB}$ value than warfarin (NCB: $0.72,95 \% \mathrm{CI}$ : $0.22-1.22, \mathrm{P}<0.01)$. Interestingly, there was an incremental $\mathrm{NCB}$ with the deterioration of renal function $(0.72$ for no CKD, 1.59 for mild CKD, 2.74 for moderate CKD, and $\mathrm{P}_{\text {interaction }}=0.001$ among different renal function). Results of sensitivity analyses, using weighted factor of 1.0 and 2.0, were consistent with those of the primacy analyses.

\section{Sensitivity analysis}

The meta-regression analysis indicated that no potential confounding was present (Table S2). When we performed the sensitivity analysis in various degrees of renal function, the results were confirmed in the mild CKD group comparing NOACs with warfarin (Figure S3). In the moderate CKD group, NOACs tended to significantly reduce the risk of SSE (RR: $0.85,95 \%$ CI: $0.72-1.00$, $\mathrm{P}=0.05$ ) and major bleeding (RR: 0.74, 95\% CI: 0.53-1.03, $\mathrm{P}=0.08$ ) compared to warfarin (Figure S4). After excluding low-dose results of RE-LY and ENGAGE AF-TIMI 48 trials in this analysis, the results were similar with the

\section{NCB of NOAC versus Warfarin}

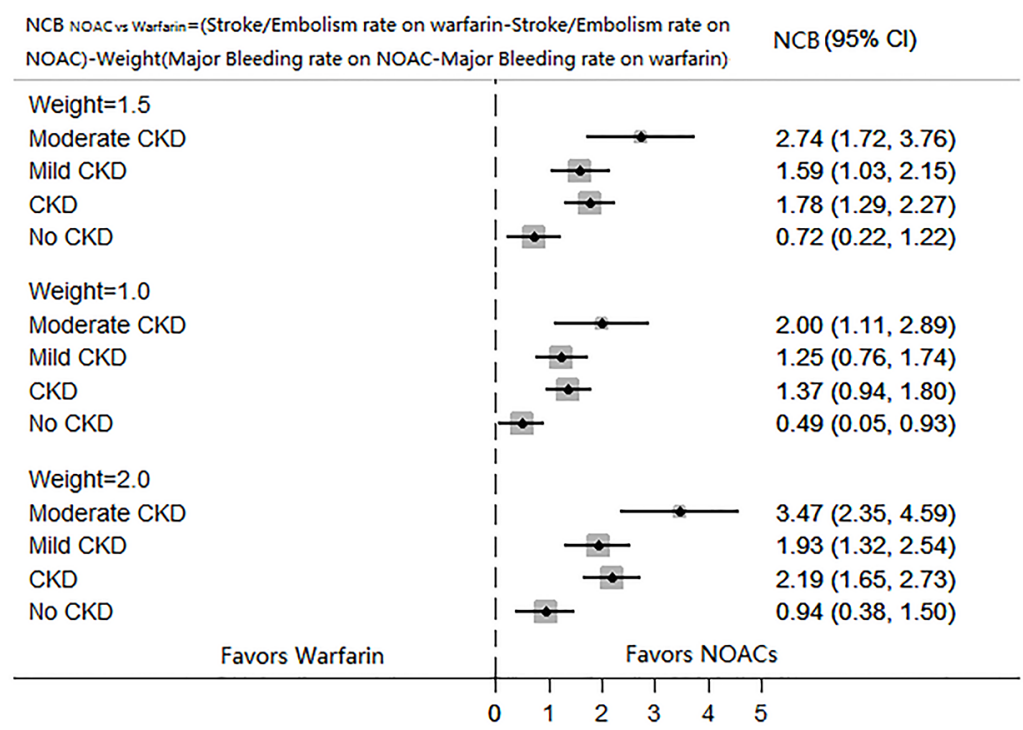

Figure $3 \mathrm{NCB}$ of NOACs versus warfarin in patients with CKD and without CKD. NCB, net clinical benefit; NOACs, non-vitamin K antagonist oral anticoagulants; CKD, chronic kidney disease; $95 \% \mathrm{CI}$, 95\% confidence interval. 
primacy analyses (Figures $S 5, S 6$ ).

\section{Publication bias}

We did not perform Funnel plot as the limited number of eligible trails (four RCTs).

\section{Discussion}

CKD has been related with higher risk for thromboembolic and bleeding occurrence, and combination of $\mathrm{CKD}$ and $\mathrm{AF}$ confers significantly greater risks $(2,3)$. NOACs provide a better efficacy and safety profile when compared with warfarin in general population. However, compared with warfarin, the NCB property of NOACs for these AF and CKD patients is limited. Because of this reason, we have assessed the efficacy, safety, and NCB of NOACs in patients with $\mathrm{AF}$ and $\mathrm{CKD}$, and have obtained the following main results. Firstly, NOACs significantly reduced the risk for SSE, major bleeding and all-cause death in patients with CKD when compared to warfarin. More importantly, NOACs, compared with warfarin, could bring about a progressively increased $\mathrm{NCB}$ value with the deterioration of renal function.

\section{Outcomes of NOACs in patients with AF and CKD}

Although CKD is not a component factor of the CHADS2 score or CHA2DS2-VASC score, it is closely associated with their risk factors, such as congestive heart failure, hypertension, age, diabetes, and prior stroke or transient ischemic attack (23). Warfarin is widely administered in AF and CKD patients due to its low-cost and availability of reversal agents. However, it is important to recognize that CKD is associated with a suboptimal TTR in AF patients treated with warfarin (3). Inadequate control of TTR might contribute to increased risk of thromboembolism as well as bleeding (24). Similarly, our results found that CKD patients receiving NOACs also brought more SSE events (1.57\%/ year versus $1.01 \%$ /year) and major bleeding $(3.13 \% /$ year versus $1.88 \%$ /year) than no-CKD patients, meaning that CKD is still a strong factor for thromboembolism and hemorrhage despite the use of NOACs. CKD progression is often characterized by fluctuant creatinine levels and acute kidney injury events that may affect renal elimination of NOACs and make patients exposed at an increased risk of hemorrhage (25). Hence, intensive caution should be proceeded with NOACs in CKD patients, including close monitoring of renal function.

\section{Outcomes of NOACs versus warfarin in CKD patients}

All the sub-analysis of included trials divided the CKD into 3 different levels based on $\mathrm{CrCl}(>80 \mathrm{~mL} / \mathrm{min}, 50$ to $80 \mathrm{~mL} / \mathrm{min}$, and $<50 \mathrm{~mL} / \mathrm{min}$ ). In RE-LY, dabigatran 150 $\mathrm{mg}$ twice daily showed a significantly decreased risk for SSE in mild and moderate CKD, and $110 \mathrm{mg}$ significantly decreased the risk for major bleeding only in patients with moderate CKD. No difference was reported between CKD and no CKD subgroup (20). In ROCKET AF, rivaroxaban $15 \mathrm{mg}$ daily in subjects with moderate CKD, did not show significant difference in comparison to warfarin regarding the SSE and major bleeding (21). In ARISTOTLE, apixaban $5 \mathrm{mg}$ twice daily was more effective than warfarin at preventing SSE and major bleeding in mild CKD (17). In ENGAGE AF-TIMI 48, edoxaban $60 \mathrm{mg}$ daily was associated with fewer SSE events in mild CKD as well as fewer major bleeding events in moderate CKD (22). Our analysis, merging all the NOACs for powerful statistics, showed that the use of NOACs was superior to warfarin for the prevention of SSE and major bleeding in CKD patients. In addition, when the low-dose arms of RE-LY and ENGAGE AF-TIMI 48 were excluded in the analysis, the results were similar. Thus, NOACs represent a preferable and valuable alternative to warfarin in this clinical setting, while these advantages were not detected in patients without CKD.

\section{NCB of NOACs versus warfarin in patients with CKD}

$\mathrm{NCB}$, which incorporates both the risk for SSE and major bleeding, provides a more quantitatively informed basis for the decision-making on the optimal anticoagulant therapy in AF patients. Our analysis showed a superior NCB property of NOACs in comparison to warfarin in patients with $\mathrm{AF}$ and CKD. Actually, patients receiving NOACs could prevent the event in around 1.8 per 100 patients with CKD. Similarly, the use of NOACs would avoid the event in around 0.7 per 100 patients without CKD. Interestingly, our analysis found that NCB was progressively increased with the deterioration of renal function $\left(\mathrm{P}_{\text {interaction }}=0.001\right)$, with the greatest $\mathrm{NCB}$ of 2.74 in moderate $\mathrm{CKD}$ patients. In the fragile population, the major contributor of these results is a significant lower event rate of major bleeding in the NOACs group versus warfarin group, yielding a rate difference of 1.47. In fact, renal dysfunction is regarded as 
component factor of HAS-BLED, the most popular score currently used for the assessment of bleeding risk (26). Additionally, using impact weights of 1.0 and 2.0 for the difference in rates of major bleeding between patients taking NOACs or warfarin did not change our estimate of NCB. Thus, based on our analysis of NCB, NOACs is preferable to warfarin for all those with $\mathrm{AF}$, and the effect is markedly greater in patients with CKD.

\section{Study limitations}

Several limitations are worth mentioning. Firstly, the included studies were not especially assigned to assess the efficacy and safety of NOACs in CKD patients. And further analysis for patient-level data associated with patient demographics, bleeding risk factors, concomitant drugs etc. were not obtained. However, we performed a meta-regression analysis to assess available potential effect modifiers in baseline characteristics, and the results failed to detect any observational confounding factor as having influenced the primacy outcome. In addition, Singer's method was used to calculate the NCB in our analysis using a weighted index of 1.5 , which cannot account for all clinical variables (13). However, we performed a sensitivity analysis using weighted factor of 1.0 and 2.0, and a similar result was obtained. In fact, currently, there are no methods available to estimate the NCB perfectly. Finally, the J-ROCKET trial, which was a phase III trial comparing rivaroxaban with warfarin, was not included in the analysis due to the small sample size and enrolling patients only in one country, leading to the possible selection bias in our analysis (27). It is important to acknowledge that our results only involve limited number of studies and therefore cannot be extrapolated to the overall population with AF and CKD, while may be used only in patients with similar characteristics included in the present analysis.

\section{Conclusions}

The use of NOACs may bring about a better profile on efficacy, safety, and NCB when compared to warfarin in CKD patients.

\section{Acknowledgments}

Funding: This work was supported by Grants from the National Science Foundation of China (No. 81700423), National Nature Science Foundation of China (No.
81803841), Research Funds of Shanghai health and family planning commission (20184Y0022), Clinical Pharmacy Innovation Research Institute of Shanghai Jiao Tong University School of Medicine (CXYJY2019ZD001), Program for Key but Weak Discipline of Shanghai Municipal Commission of Health and Family Planning (2016ZB0304), Clinical Research Innovation and Cultivation Fund of Renji Hospital (No. PYIII-17-003), Shanghai Outstanding Young Doctor Training Program from Shanghai Municipal Commission of Health and Family Planning (to SF Yang), Shanghai Jiaotong University Medical Engineering Cross Fund (No. YG2016QN57), Scientific research project of Shanghai municipal commission of health and family planning (No. 20164Y0058).

\section{Footnote}

Conflicts of Interest: The authors have no conflicts of interest to declare.

Ethical Statement: The authors are accountable for all aspects of the work in ensuring that questions related to the accuracy or integrity of any part of the work are appropriately investigated and resolved.

\section{References}

1. Olesen JB, Lip GY, Kamper AL, et al. Stroke and bleeding in atrial fibrillation with chronic kidney disease. $\mathrm{N} \mathrm{Engl} \mathrm{J}$ Med 2012;367:625-35.

2. Alonso A, Lopez FL, Matsushita, et al. Chronic kidney disease is associated with the incidence of atrial fibrillation: the Atherosclerosis Risk in Communities (ARIC) study. Circulation 2011;123:2946-53.

3. Chan KE, Giugliano RP, Patel MR, et al. Nonvitamin K Anticoagulant Agents in Patients With Advanced Chronic Kidney Disease or on Dialysis With AF. J Am Coll Cardiol 2016;67:2888-99.

4. Gu ZC, Zhou LY, Shen L, et al. Non-vitamin K Antagonist Oral Anticoagulants vs. Warfarin at Risk of Fractures: A Systematic Review and Meta-Analysis of Randomized Controlled Trials. Front Pharmacol 2018;9:348.

5. Chao TF, Chiang CE, Lin YJ, et al. Evolving Changes of the Use of Oral Anticoagulants and Outcomes in Patients With Newly Diagnosed Atrial Fibrillation in Taiwan. Circulation 2018;138:1485-7.

6. Desai NR, Krumme AA, Schneeweiss S, et al. Patterns of initiation of oral anticoagulants in patients with atrial 
fibrillation-quality and cost implications. Am J Med 2014;127:1075-82.

7. Pelliccia F, Rosanio S, Marazzi G, et al. Efficacy and safety of novel anticoagulants versus vitamin $\mathrm{K}$ antagonists in patients with mild and moderate to severe renal insufficiency: Focus on apixaban. Int J Cardiol 2016;225:77-81.

8. McCullough PA, Ball T, Cox KM, et al. Use of Oral Anticoagulation in the Management of Atrial Fibrillation in Patients with ESRD: Pro. Clin J Am Soc Nephrol 2016;11:2079-84.

9. Nielsen PB, Lane DA, Rasmussen LH, et al. Renal function and non-vitamin $\mathrm{K}$ oral anticoagulants in comparison with warfarin on safety and efficacy outcomes in atrial fibrillation patients: a systemic review and metaregression analysis. Clin Res Cardiol 2015;104:418-29.

10. Andò G, Capranzano P. Non-vitamin K antagonist oral anticoagulants in atrial fibrillation patients with chronic kidney disease: A systematic review and network metaanalysis. Int J Cardiol 2017;231:162-9.

11. Sardar P, Chatterjee S, Herzog E, et al. Novel oral anticoagulants in patients with renal insufficiency: a metaanalysis of randomized trials. Can J Cardiol 2014;30:888-97.

12. Del-Carpio Munoz F, Gharacholou SM, Munger TM, et al. Meta-Analysis of Renal Function on the Safety and Efficacy of Novel Oral Anticoagulants for Atrial Fibrillation. Am J Cardiol 2016;117:69-75.

13. Singer DE, Chang Y, Fang MC, et al. The net clinical benefit of warfarin anticoagulation in atrial fibrillation. Ann Intern Med 2009;151:297-305.

14. Watanabe E, Yamamoto M, Kodama I, et al. Net clinical benefit of adding aspirin to warfarin in patients with atrial fibrillation: Insights from the J-RHYTHM Registry. Int J Cardiol 2016;212:311-7.

15. Chan PH, Huang D, Lau CP, et al. Net Clinical Benefit of Dabigatran Over Warfarin in Patients With Atrial Fibrillation Stratified by CHA2DS2-VASc and Time in Therapeutic Range. Can J Cardiol 2016;32:1247.

16. Bonde AN, Lip GY, Kamper AL, et al. Net clinical benefit of antithrombotic therapy in patients with atrial fibrillation and chronic kidney disease: a nationwide observational cohort study. J Am Coll Cardiol 2014;64:2471-82.

17. Hohnloser SH, Hijazi Z, Thomas L, et al. Efficacy of apixaban when compared with warfarin in relation to renal function in patients with atrial fibrillation: insights from the ARISTOTLE trial. Eur Heart J 2012;33:2821-30.

18. Yan YD, Zhang C, Shen L, et al. Net Clinical Benefit of Non-vitamin K Antagonist Oral Anticoagulants for Venous Thromboembolism Prophylaxis in Patients With
Cancer: A Systematic Review and Trade-Off Analysis From 9 Randomized Controlled Trials. Front Pharmacol 2018;9:575.

19. Wei AH, Gu ZC, Zhang C, et al. Increased risk of myocardial infarction with dabigatran etexilate: fact or fiction? A critical meta-analysis of over 580,000 patients from integrating randomized controlled trials and realworld studies. Int J Cardiol 2018;267:1-7.

20. Hijazi Z, Hohnloser SH, Oldgren J, et al. Efficacy and safety of dabigatran compared with warfarin in relation to baseline renal function in patients with atrial fibrillation: a RE-LY (Randomized Evaluation of Longterm Anticoagulation Therapy) trial analysis. Circulation 2014;129:961-70.

21. Fox KA, Piccini JP, Wojdyla D, et al. Prevention of stroke and systemic embolism with rivaroxaban compared with warfarin in patients with non-valvular atrial fibrillation and moderate renal impairment. Eur Heart J 2011;32:2387-94.

22. Bohula EA, Giugliano RP, Ruff CT, et al. Impact of Renal Function on Outcomes With Edoxaban in the ENGAGE AF-TIMI 48 Trial. Circulation 2016;134:24-36.

23. Uchiyama S, Hori M, Matsumoto M, et al. Net clinical benefit of rivaroxaban versus warfarin in Japanese patients with nonvalvular atrial fibrillation: a subgroup analysis of J-ROCKET AF. J Stroke Cerebrovasc Dis 2014;23:1142-7.

24. Apostolakis S, Sullivan RM, Olshansky B, et al. Factors affecting quality of anticoagulation control among patients with atrial fibrillation on warfarin: the SAMe-TT(2)R(2) score. Chest 2013;144:1555-63.

25. Di Lullo L, Ronco C, Cozzolino M, et al. Nonvitamin $\mathrm{K}$-dependent oral anticoagulants (NOACs) in chronic kidney disease patients with atrial fibrillation. Thromb Res 2017;155:38-47.

26. Kirchhof P, Benussi S, Kotecha D, et al. 2016 ESC Guidelines for the management of atrial fibrillation developed in collaboration with EACTS. Europace 2016;18:1609-78.

27. Hori M, Matsumoto M, Tanahashi N, et al. Rivaroxaban vs. warfarin in Japanese patients with atrial fibrillation the J-ROCKET AF study. Circ J 2012;76:2104-11.

Cite this article as: Gu ZC, Kong LC, Yang SF, Wei AH, Wang N, Ding Z, Zhang C, Liu XY, Zheng YL, Lin HW. Net clinical benefit of non-vitamin $\mathrm{K}$ antagonist oral anticoagulants in atrial fibrillation and chronic kidney disease: a trade-off analysis from four phase III clinical trials. Cardiovasc Diagn Ther 2019;9(5):410-419. doi: 10.21037/cdt.2019.07.09 


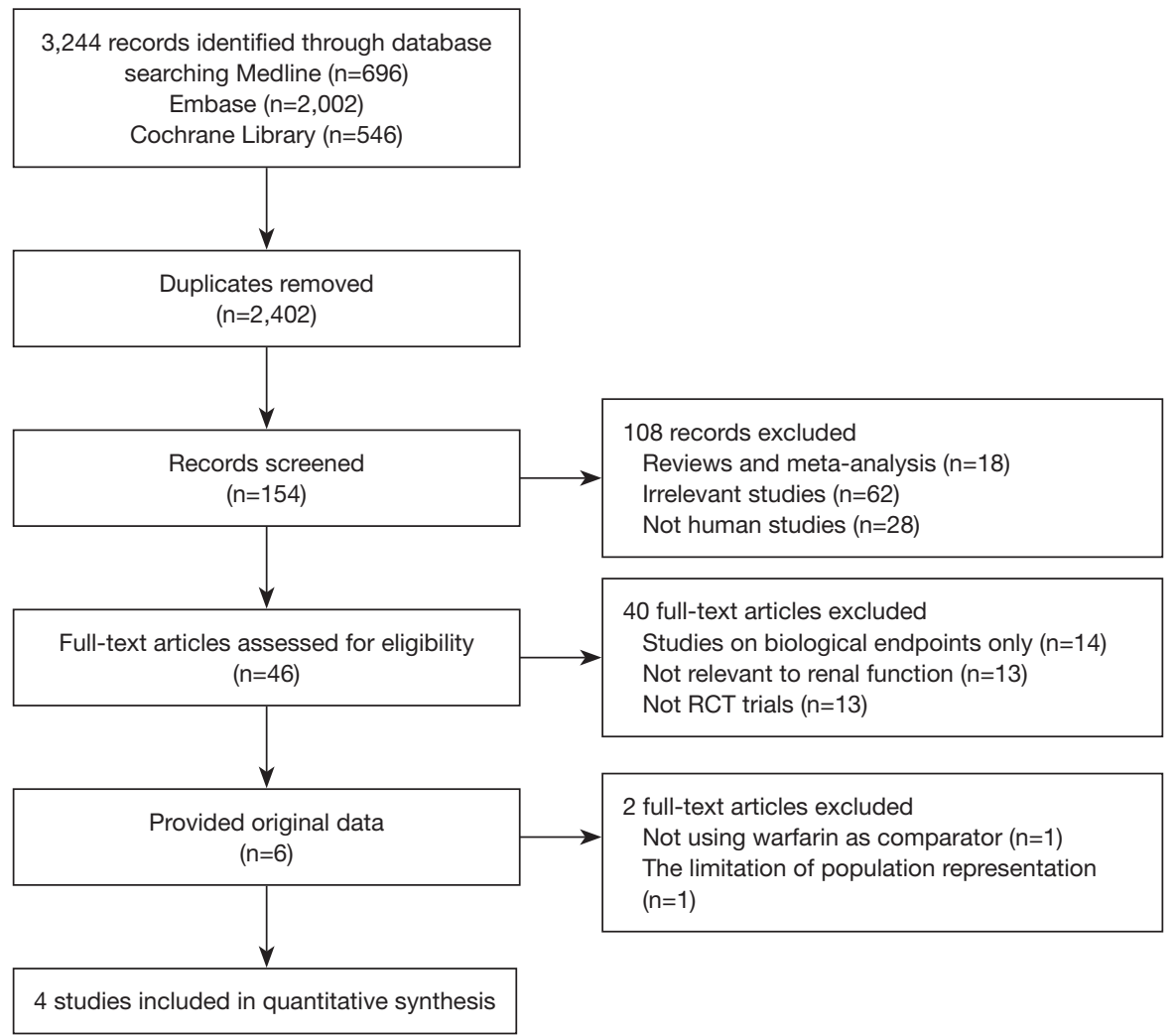

Figure S1 Flow diagram for the selection of eligible randomized controlled trials.

Table S1 Quality assessment of included trials

\begin{tabular}{|c|c|c|c|c|c|c|c|c|}
\hline Study & $\begin{array}{l}\text { Random } \\
\text { sequence } \\
\text { generation }\end{array}$ & $\begin{array}{c}\text { Allocation } \\
\text { concealment }\end{array}$ & $\begin{array}{l}\text { Blinding of } \\
\text { participants } \\
\text { and personnel }\end{array}$ & $\begin{array}{l}\text { Blinding of } \\
\text { outcome } \\
\text { assessment }\end{array}$ & $\begin{array}{l}\text { Incomplete } \\
\text { outcome } \\
\text { data }\end{array}$ & $\begin{array}{l}\text { Selective } \\
\text { reporting }\end{array}$ & $\begin{array}{l}\text { Other } \\
\text { bias }\end{array}$ & $\begin{array}{c}\text { Summary } \\
\text { risk }\end{array}$ \\
\hline ROCKET AF (21) & L & L & L & L & $\mathrm{L}$ & $U$ & L & L \\
\hline ARISTOTLE (17) & L & L & $\mathrm{L}$ & L & $\mathrm{L}$ & $U$ & L & L \\
\hline
\end{tabular}

$\mathrm{L}$, low risk; $\mathrm{U}$, unclear risk; $\mathrm{H}$, high risk. 


\section{P-value for Interaction Between CKD and No CKD}

\begin{tabular}{|c|c|c|c|}
\hline Outcome & & $\mathrm{RR}(95 \% \mathrm{Cl})$ & $P$ for interaction \\
\hline \multicolumn{4}{|c|}{ Stroke/Embolism } \\
\hline CKD & $\infty$ & $0.80(0.73,0.88)$ & \\
\hline No CKD & & $0.99(0.75,1.32)$ & $p=0.206$ \\
\hline \multicolumn{4}{|c|}{ Major Bleeding } \\
\hline CKD & $\rightarrow$ & $0.79(0.66,0.96)$ & \\
\hline No CKD & $\longrightarrow$ & $0.80(0.58,1.09)$ & $p=0.947$ \\
\hline \multicolumn{4}{|c|}{ ALL-cause Death } \\
\hline CKD & $\leftarrow$ & $0.91(0.84,0.99)$ & \\
\hline No CKD & $\rightarrow$ & $0.87(0.74,1.01)$ & $p=0.612$ \\
\hline
\end{tabular}

Figure S2 P value for interaction between patients with and without CKD. CKD, chronic kidney disease.

Table S2 Meta-regression analysis

\begin{tabular}{|c|c|c|}
\hline Variable & Stroke/embolism (P value/tau²) & Major bleeding ( $\mathrm{P}$ value/tau $\left.{ }^{2}\right)$ \\
\hline \multicolumn{3}{|l|}{ All cohort } \\
\hline Age & $0.777 / 0.026$ & $0.114 / 0.010$ \\
\hline Female & $0.608 / 0.017$ & $0.214 / 0.021$ \\
\hline $\mathrm{CHF}$ & $0.260 / 0.000$ & $0.871 / 0.060$ \\
\hline Hypertension & $0.270 / 0.000$ & $0.621 / 0.052$ \\
\hline Diabetes & $0.261 / 0.000$ & $0.856 / 0.060$ \\
\hline Stroke or TIA & $0.528 / 0.001$ & $0.326 / 0.031$ \\
\hline Aspirin & $0.430 / 0.000$ & $0.200 / 0.019$ \\
\hline VKA & $0.759 / 0.020$ & $0.476 / 0.043$ \\
\hline \multicolumn{3}{|l|}{ CKD } \\
\hline Age & $0.937 / 0.000$ & $0.670 / 0.019$ \\
\hline Female & $0.988 / 0.000$ & $0.332 / 0.006$ \\
\hline $\mathrm{CHF}$ & $0.912 / 0.000$ & $0.792 / 0.046$ \\
\hline Hypertension & $0.953 / 0.000$ & $0.597 / 0.046$ \\
\hline Diabetes & $0.860 / 0.000$ & $0.894 / 0.031$ \\
\hline Stroke or TIA & $0.866 / 0.000$ & $0.941 / 0.027$ \\
\hline Aspirin & $0.926 / 0.000$ & $0.172 / 0.006$ \\
\hline VKA & $0.844 / 0.000$ & $0.477 / 0.021$ \\
\hline \multicolumn{3}{|l|}{ No CKD } \\
\hline Age & $0.365 / 0.058$ & $0.637 / 0.018$ \\
\hline Female & $0.331 / 0.072$ & $0.602 / 0.006$ \\
\hline $\mathrm{CHF}$ & $0.216 / 0.029$ & $0.290 / 0.134$ \\
\hline Hypertension & $0.382 / 0.020$ & $0.654 / 0.134$ \\
\hline Diabetes & $0.214 / 0.000$ & $0.306 / 0.133$ \\
\hline Stroke or TIA & $0.789 / 0.098$ & $0.493 / 0.038$ \\
\hline Aspirin & $0.486 / 0.036$ & $0.762 / 0.102$ \\
\hline VKA & $0.898 / 0.097$ & $0.606 / 0.134$ \\
\hline
\end{tabular}

$P$ values: it is the results of meta-regression for the relationship between each variable and the outcomes; CHF, congestive heart failure; TIA, transient ischemic attack; VKA, vitamin $\mathrm{K}$ antagonist. 
NOAC versus Warfarin in Mild CKD

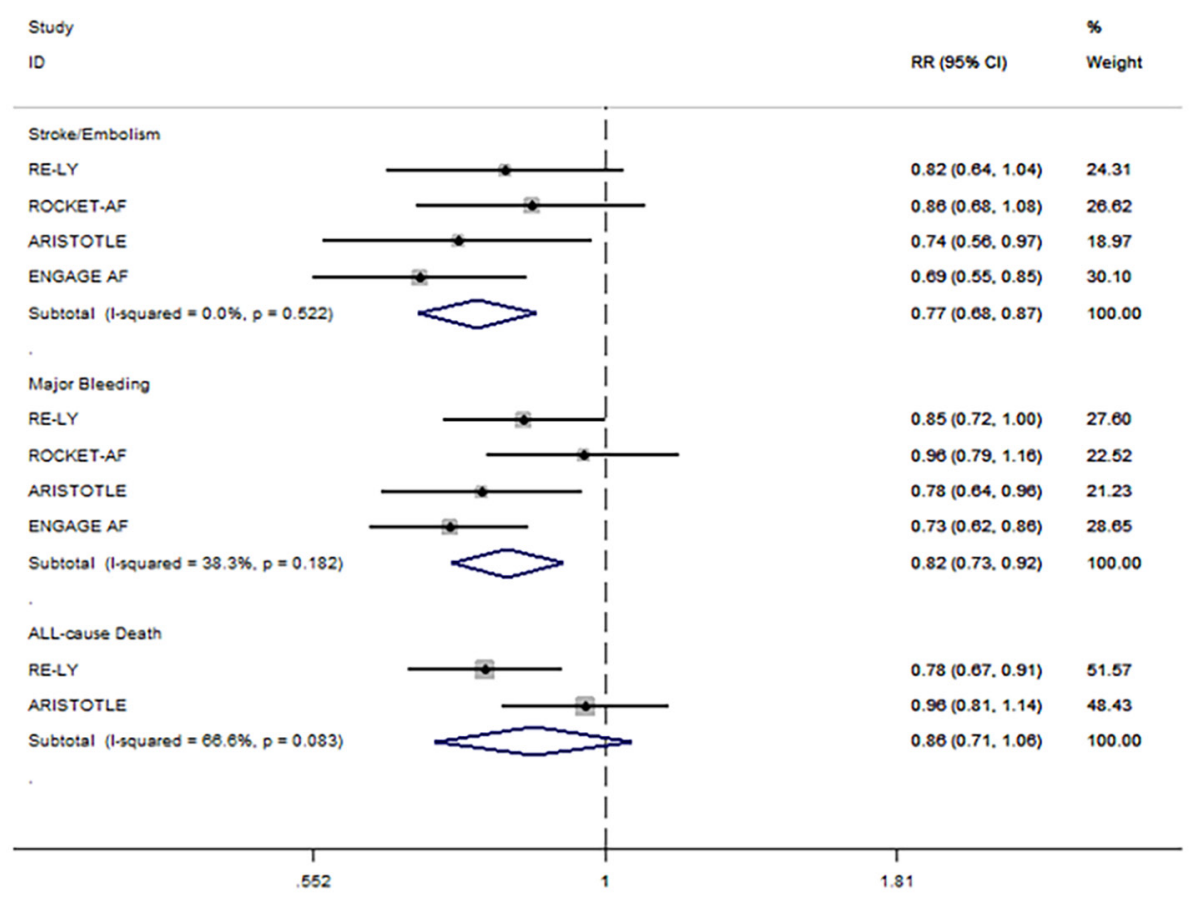

Figure S3 Sensitivity analysis of NOAC versus warfarin in mild CKD. NOAC, non-vitamin K antagonist oral anticoagulant; CKD, chronic kidney disease.

\section{NOAC versus Warfarin in Moderate CKD}

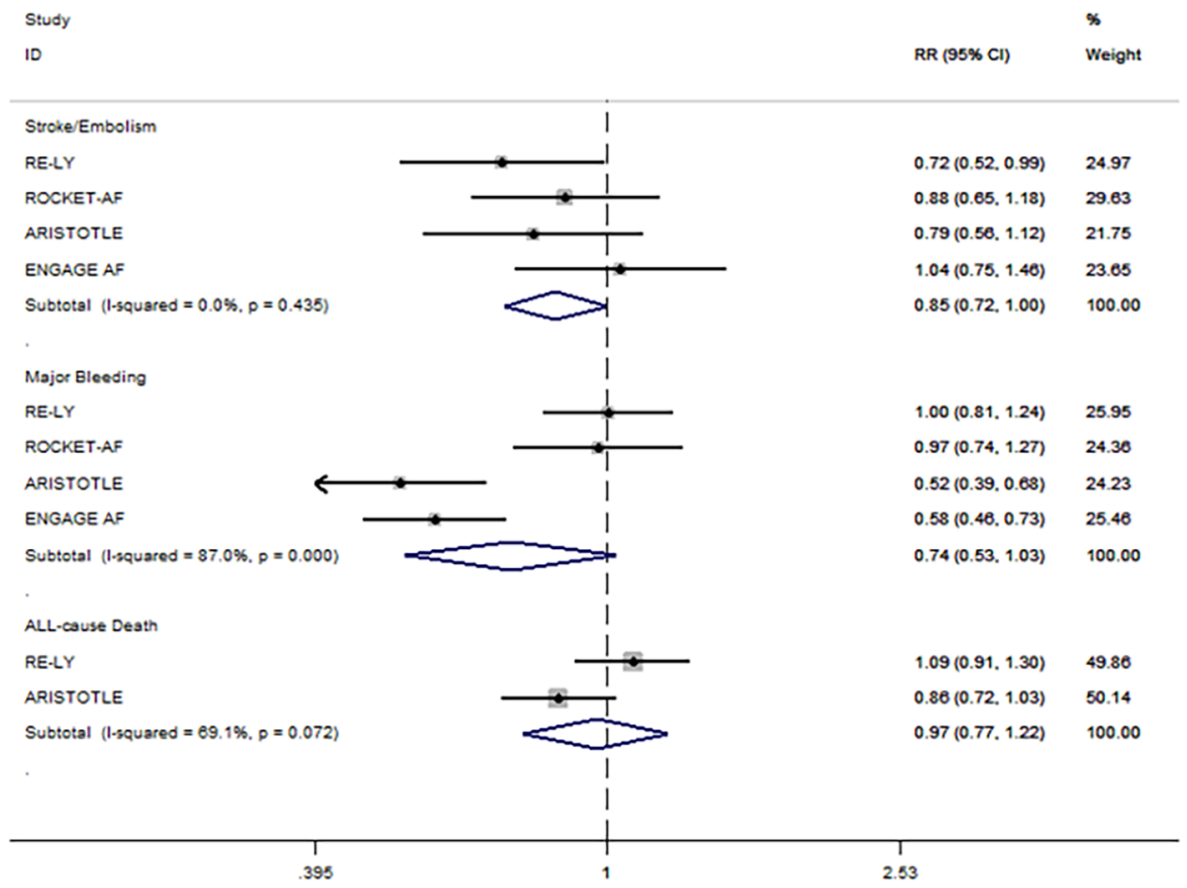

Figure S4 Sensitivity analysis of NOAC versus warfarin in moderate CKD. NOAC, non-vitamin K antagonist oral anticoagulant; CKD, chronic kidney disease. 
NOAC versus Warfarin in CKD (High-dose Rigimen)

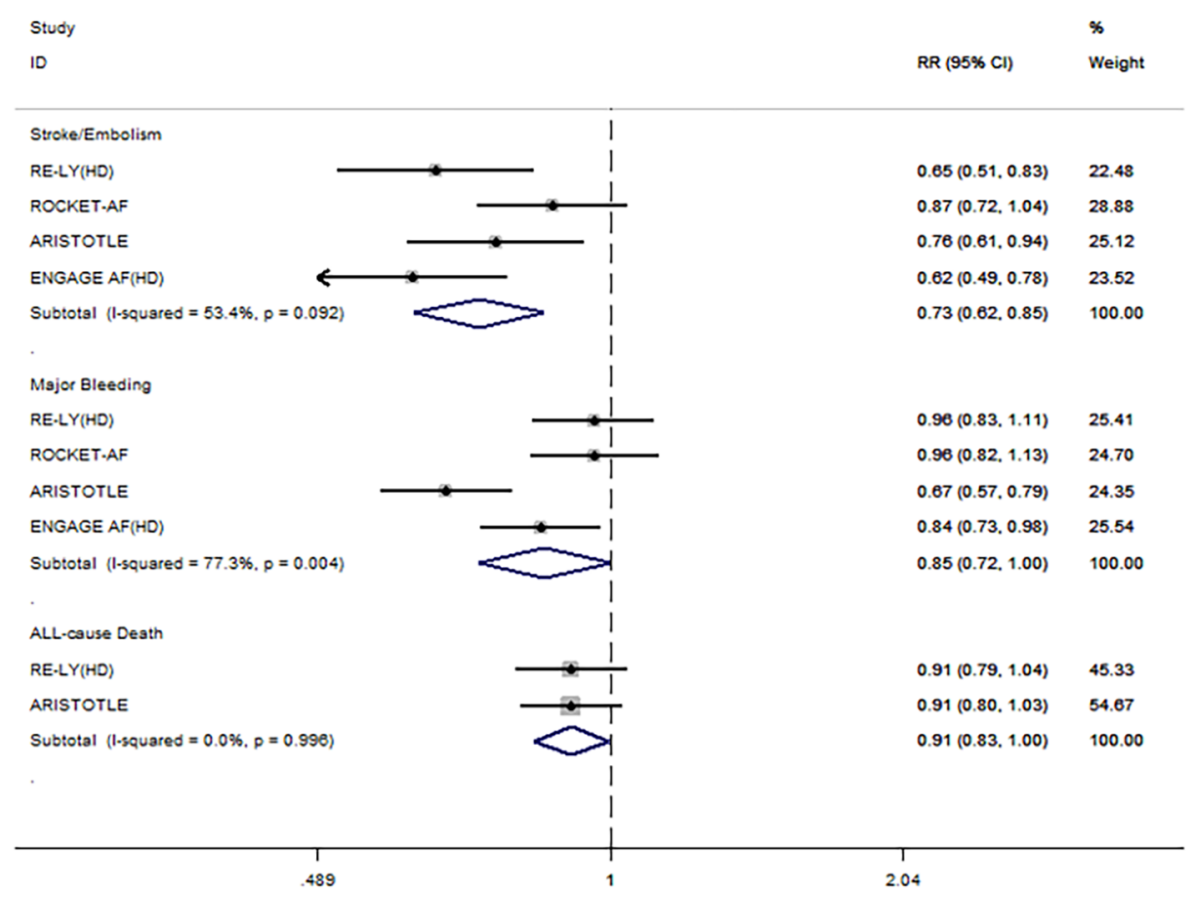

Figure S5 Sensitivity analysis of NOAC versus warfarin in CKD after removing the low-dose regimen. NOAC, non-vitamin K antagonist oral anticoagulant; CKD, chronic kidney disease.

NOAC versus Warfarin in No CKD (High-dose Regimen)

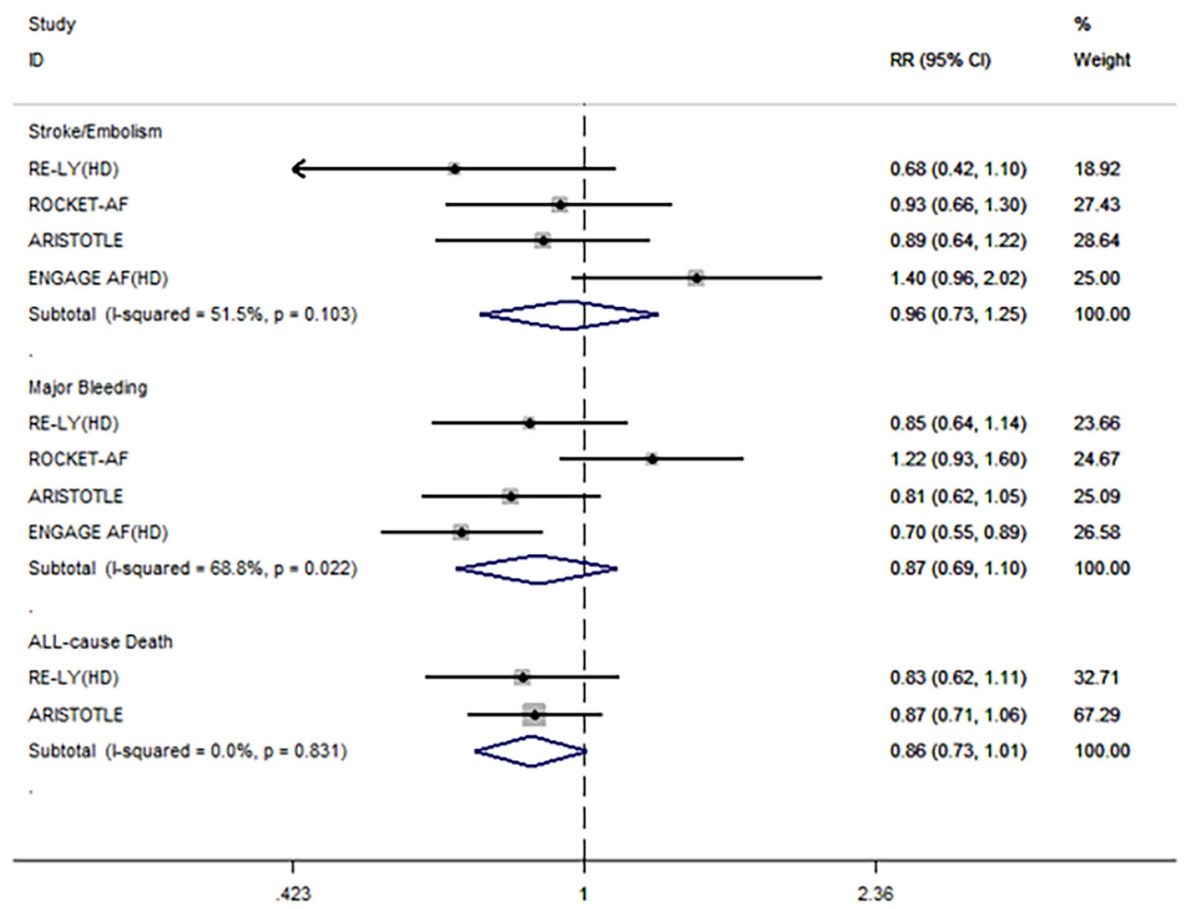

Figure S6 Sensitivity analysis of NOAC versus warfarin in no CKD after removing the low-dose regimen. NOAC, non-vitamin K antagonist oral anticoagulant; $\mathrm{CKD}$, chronic kidney disease. 